\title{
Objective Assessment of Impulse Control Disorder in Patients with Parkinson's Disease Using a Low- Cost LEGO-Like EEG Headset
}

\author{
Yuan-Pin Lin \\ National Sun Yat-sen University \\ Hsing-Yi Liang \\ National Sun Yat-sen University \\ Yueh-Sheng Chen \\ Kaohsiung Chang Gung Memorial Hospital \\ Cheng-Hsien Lu \\ Kaohsiung Chang Gung Memorial Hospital \\ Yih-Ru Wu \\ Linkou Chang Gung Memorial Hospital

\section{Yung-Yee Chang} \\ Kaohsiung Chang Gung Memorial Hospital \\ Wei-Che Lin ( $\nabla$ alex@cgmh.org.tw ) \\ Kaohsiung Chang Gung Memorial Hospital
}

\section{Research}

Keywords: Parkinson's disease, Impulse control disorders, Electroencephalogram, Event-related potential, LEGO-like headset

Posted Date: December 11th, 2020

DOI: https://doi.org/10.21203/rs.3.rs-125029/v1

License: (c) (1) This work is licensed under a Creative Commons Attribution 4.0 International License. Read Full License 


\title{
Objective Assessment of Impulse Control Disorder in Patients with Parkinson's Disease Using a Low- Cost LEGO-like EEG Headset
}

\author{
1,2 Yuan-Pin Lin (yplin@mail.nsysu.edu.tw) \\ ${ }^{1}$ Hsing-Yi Liang (hy@imst.nsysu.edu.tw) \\ ${ }^{3}$ Yueh-Sheng Chen (qqqqqq4545@cgmh.org.tw) \\ ${ }^{4}$ Cheng-Hsien Lu (chlu99@cgmh.org.tw) \\ ${ }^{5}$ Yih-Ru Wu (yihruwu@cgmh.org.tw) \\ ${ }^{4}$ Yung-Yee Chang (changyy7@gmail.com) \\ ${ }^{3}$ Wei-Che Lin (alex@cgmh.org.tw)
}

${ }^{1}$ Institute of Medical Science and Technology, National Sun Yat-sen University, Kaohsiung, Taiwan

${ }^{2}$ Department of Electrical Engineering, National Sun Yat-sen University, Kaohsiung, Taiwan

${ }^{3}$ Department of Diagnostic Radiology, Kaohsiung Chang Gung Memorial Hospital, and Chang Gung University College of Medicine, Kaohsiung, Taiwan

${ }^{4}$ Department of Neurology, Kaohsiung Chang Gung Memorial Hospital, and Chang Gung University College of Medicine, Kaohsiung, Taiwan, Kaohsiung, Taiwan

${ }^{5}$ Department of Neurology, Linkou Chang Gung Memorial Hospital, and Chang Gung University College of Medicine, Taoyuan, Taiwan

\section{Correspondence: Dr. Wei-Che Lin}

\section{Address:}

Department of Diagnostic Radiology,

Kaohsiung Chang Gung Memorial Hospital,

No. 123, Dapi Road, Niaosong District, Kaohsiung City, 833, Taiwan

Phone: +886-7-731-7123 ext. 3050

Email: alex@cgmh.org.tw 


\begin{abstract}
Background: Patients with Parkinson's disease (PD) can develop the cognitive adverse effect of impulse control disorders (ICDs) while undergoing a pharmacological treatment for motor control dysfunctions with a dopamine agonist (DA). Conventional clinical interviews or questionnaires can be biased and may not provide an accurate diagnosis in the early stage. A wearable electroencephalogram (EEG)-sensing headset paired with an examination procedure can be a potential user-friendly method to explore ICD-related biomarkers that can reflect brain activity abnormalities and detect its early signs and progression.
\end{abstract}

Methods: A stereotypical Go/NoGo test that targets impulse inhibition was performed with 59 individuals, including heathy controls, patients with PD, and patients with PD diagnosed with ICD. A low-cost LEGO-like EEG headset was used to record concurrent EEG signals. The event-related potential (ERP) analytical framework was then used to explore ICD-related EEG abnormalities after DA treatment.

Results: Only PD patients with ICD exhibited a tendency for N2 and P3 amplitude deterioration at the fronto-central regions (i.e., $\mathrm{Fz}, \mathrm{FCz}$, and $\mathrm{Cz}$ ); in particular, the $\mathrm{P} 3$ counterpart reached statistical significance $(p<0.05)$. Neither PD patients nor healthy controls (without DA) replicated such findings. Furthermore, N2 amplitude deterioration was found to be related to ICD severity at Fz $(r=-0.28, p=0.04)$.

Conclusions: A low-cost LEGO-like EEG headset successfully captured ERP neuromarkers for the objective assessment of ICD in PD patients undergoing DA treatment. The present objective neuro-evidence could provide complementary information to conventional clinical scales used to diagnose the ICD adverse effect.

Keywords: Parkinson's disease, Impulse control disorders, Electroencephalogram, Eventrelated potential, LEGO-like headset 


\section{Background}

Parkinson's disease (PD) is a progressive neurodegenerative disorder characterized by the loss of midbrain dopaminergic neurons and the subsequent depletion of dopamine levels in the basal ganglia [1]. PD patients manifest the hallmarks of motor control dysfunction, i.e., tremor, bradykinesia, and rigidity. Moreover, the disorder is frequently accompanied by a cognitive decline [2,3] in many aspects, e.g., behavioral response, attention shift, reward learning, and working memory. Particularly, the main pharmacological treatment for the motor symptoms, i.e., dopamine agonists (DA), may trigger impulse control disorders (ICDs) as an adverse effect [4-8]. ICD refers to the inability to inhibit processes, thereby leading to several compulsive or pathological disorders related to gambling, shopping, eating, and sexuality [4]. The inhibitory control is the capability of selecting the most appropriate response while suppressing competing counterparts in ever-changing circumstances. The integrity of impulse control is critical for controlling behavior at all levels in real life [4, 8]. Thus, assessing, monitoring, and, ideally, avoiding the DA-associated adverse effect of ICD in PD patients has become increasingly importance [4, 7-9].

Noteworthily, the ICD adverse effect can be mitigated and even terminated by reducing the DA dose or switching to another dopamine-replacement therapy [4]. At present, assessing the presence of ICD mainly relies on subjective clinical judgement associated to interview outcomes of PD patients, as well as their self-reported questionnaire scores. However, behavioral scales could be biased and may not provide an accurate diagnosis at early stages. The recent advances in neuroimaging benefit the exploration of impulse control-relevant neural networks and of their interaction with psychopharmacological interventions. Several neuroimaging techniques, including near-infrared spectroscopy [10], functional magnetic resonance imaging $[11,12]$, and electroencephalogram (EEG) $[13,14]$ have demonstrated the feasibility of associating neurological evidence with inhibitory control. Thus, it is possible to find brain-markers that objectively characterize cognitive decline in neurodegenerative diseases [15], for example, impulse control integrity in PD patients during chronic DA treatment.

Among the available neuroimaging techniques, EEG measures the electrical brain activity with a high temporal resolution of milliseconds, which enables capturing the onset of cognitive states and their rapid transitions. In addition, wearable EEG-recording technology has made impressive progress in recent years. Unlike the bulky gel electrode-headset of laboratories, wearable technology allows the recording of brain activity using dry/saline electrodes, wireless transmission, and a minimized amplifier [16-18]. Furthermore, the easy-to-setup wearability 
not only makes the EEG measurement more user/patient-friendly and less headset-calibrated, but also considerably promotes realistic EEG applications in daily life [19-21].

Event-related potential (ERP) is one of the well-established signaling markers related to qualitative and quantitative assessment of cognitive processes. During EEG recording, the individual undergoes a task-specific experimental protocol to study a cognitive capacity of interest for sequential ERP analysis. Previous studies have linked the alteration of ERP waveforms to the integrity of the targeted cognitive function. For example, the oddball paradigm is a classic task to engage the selective attention network. When the brain perceives a deviant yet target stimulus, the P3 component (a positive peak around 300-500 ms after stimulus onset) presents dominantly at the midline scalp electrodes [22, 23]. An attenuated or absent P3 component could implicate alterations or even deficits in attention shifting [3, 24]. The Go/NoGo task is another common task to investigate cognitive and motoric inhibition [25, 26]. While frequent Go trials are proceeded by an as-fast-as-possible behavioral response, the rarely presented NoGo trials imply a withholding of the prepotent response, i.e., inhibition control. The successful NoGo inhibition normally leads to apparent N2 (negativity around 200 $300 \mathrm{~ms}$ ) and P3 signals at fronto-central regions as compared to Go trials [13, 25, 26]. Analogously to the abovementioned, diminished amplitudes of N2 and P3 components have been associated to dysfunction in inhibition control in individuals with attentiondeficit/hyperactivity disorder [27], internet addiction [28], and PD [14]. Accordingly, the ERP signature during an actively engaged cognitive task is well suited to examine the deficits in the targeted cognitive function in physiological, psychological, and psychiatric disorders.

Motivated by the ERP assessment of cognitive capacity and its applicability to Go/NoGo task-engaged inhibition control, the use of ERP signaling strategy to reveal ICD markers in PD patients undergoing DA pharmacological interventions was attempted in this work. This work followed the hypothesis that the PD patients diagnosed with ICD would exhibit diminished amplitudes of the N2 and P3 complex after DA treatment as compared to typical PD patients. This amplitude degradation would be related to ICD severity. That is, the more severe the ICD symptoms, the more diminished the amplitude. A further characteristic of the present work was to approach the above issue using a customized, cost-efficient EEG amplifier [29] and electrode-holder assembly [30] (namely LEGO-like EEG headset). The use of a nonmedicalgrade EEG-sensing platform is a harsh yet realistic setup, closer to a practical wearable-device for ubiquitous ICD monitoring. While most previous studies focused on the EEG/ERP distinction between PD patients and healthy subjects $[14,31,32]$ or between different PD stages 
[33-35], the present work attempted to address whether ERP signatures can be regarded as neuro-markers reflecting the DA-triggered ICD adverse effect in PD patients. This work also contributes to the practical application of EEG by using a customized, cost-efficient EEGsensing setup rather than an expense laboratory-oriented product. Successful results will elucidate how an EEG-based wearable device can monitor ICD risk in each PD patient at home routinely, and how it may, thereby, guide clinical practice to an optimal DA dose management or pharmacological plan while treating motor symptoms.

\section{Methods}

\subsection{Participants}

This study recruited 59 subjects who were divided into three groups of 23 PD patients (16 males, 7 females, mean age \pm standard deviation: 64.30 \pm 9.56 years; named PD group hereafter), 10 PD patients with ICD ( 8 males, 2 females; mean age 63.22 \pm 7.74 years; named ICD group hereafter), and 26 healthy controls (13 males and 13 females; mean age 59.26 \pm 6.85 years; named HC group hereafter). Each subject filled the questionnaire for impulsive-compulsive disorders in Parkinson's disease-rating scale (QUIP-RS) [36] before the experiment. The QUIP$\mathrm{RS}$ is a valid and reliable rating scale for ICD and is useful in monitor its severity over time. The subjects' self-reported QUIP-RS scores were used to explore associations with the ERP signatures. Age did not statistically differ between three groups $(p>0.05)$, but the QUIP-RS score was significantly higher $(p<0.01)$ in the ICD group than in the PD and HC groups (ICD: 16.00 \pm 12.32 , PD: $0.45 \pm 1.15$, HC: 0 ). The statistical assessment of HC vs. PD was performed with the two-sample $t$-test, whereas HC/PD vs. ICD were assessed with the Wilcoxon rank-sum test due to the imbalance in group samples. The clinical assessment of subjects with PD/ICD and their data collection took place in Kaohsiung Chang Gung Memorial Hospital (CGMH). The study protocol was approved by the CGMH ethics committee. Each subject was fully informed about the research purpose and provided written consent prior to the experiment. No subjects have experienced the employed experimental task previously. Neither the DA dose nor the treatment were changed for any PD patient as per their EEG analytical outcome.

\subsection{Go/NoGo Experiment and EEG Signal Collection}

A two-target visual Go/NoGo task was selected to elicit cognitive and motoric inhibition during the EEG recording. The subject had to press a handheld button as quickly and accurately as possible on presentation of a green square (i.e., Go trials, $8.5 \mathrm{~cm} \times 8.5 \mathrm{~cm}$ ), which was frequently presented (70\%), at the center of a 16" monitor, but withhold from button-pressing 
on presentation of a red square (i.e., NoGo trial), which was rarely presented (30\%). The protocol is shown in Figure 1(A). Each subject underwent the Go/NoGo task in two sessions with an $\sim 1 \mathrm{~h}$ inter-session rest. PD and ICD subjects took their DA medication right after completing the $1^{\text {st }}$ session. Each session was composed of three 80-trial blocks, which last around 30 minutes. The inter-trial jitter was set to $0.5-1.5$ seconds. Each session collected a total of 168 Go trials and 72 NoGo trials per subject.

This study used a lab-customized, cost-efficient, and potable 8-channel EEG amplifier [29] and wired it to a LEGO-like electrode-holder assembly [30]. The amplifier sampled the EEG signals at $250 \mathrm{~Hz}$ and in a bandwidth of $0.6-56.5 \mathrm{~Hz}$. To each set of the LEGO headset (i.e., sensor positioning ring, inter-ring bridge, and bridge shield), an 8-channel electrode-holder grid was assembled to position the EEG electrodes over the locations of F3, Fz, F4, FCz, C3, Cz, $\mathrm{C} 4$, and $\mathrm{CPz}$ (see Figure 1(B)), mainly covering the fronto-central region relevant to the inhibition processing of N2 and P3 components [13, 25, 26]. The assembled LEGO headset accommodated the dry electrodes (Cognionics Inc., San Diego, CA) for the measurement with respect to left and right earlobes as ground and reference sites, respectively. Regarding the integrity of the customized EEG recording infrastructure, the portable amplifier has been demonstrated to record ERP P3 waveforms in an auditory oddball paradigm using a hyperscanning setup for three subjects with a 10-day reproducibility test [29]. Its integration to the LEGO headset has been also verified by not only a steady-state visual-evoked potential (SSVEP) task [30], but also by the same oddball task with still and walking subjects [30, 37]. Please refer to [29, 30] for more details about the portable amplifier and LEGO headset designs. Figure $1(\mathrm{C})$ presents the experimental setup for the Go/NoGo task and the EEG recording.

\subsection{Signal Preprocessing and Analysis}

This study adopted the following procedures to pre-process the collected EEG signals and extract N2 and P3 peak amplitudes corresponding to the Go and NoGo trials per EEG session. Firstly, the raw EEG signals were band-pass filtered into a bandwidth of 1-30 Hz. The filtered EEG signals were then segmented into epochs from -200 to $1000 \mathrm{~ms}$, as per the visual target onset, and corrected upon their pre-stimulus baseline. The artifactual epochs with a statistical kurtosis value exceeding a threshold of 4 were rejected, followed by a visual inspection to ensure signal quality. In addition, the epochs corresponding to erroneous behavioral responses (standard error: no button-pressing for Go trials, commission error: button-pressing for NoGo trials) were further discarded. As such, there were seven subjects (HC: 3, PD: 3, ICD: 1) whose retained epochs (less than $80 \%$ ) were discarded due to either technical or personal issues. The 
remaining 52 subjects retained $\sim 91 \%$ epochs on average for sequential analysis.

This work employed an independent component analysis (ICA) to remove eye movement artifacts that commonly accompany a visual task. The remaining epochs were incorporated to an extended infomax ICA algorithm to parse the 8-channel signals into independent components (ICs). ICs with pronounced characteristics of eye movement in terms of activation waveform and spectral profile were identified and removed [38]. The remaining ICs were then back-projected to the channel-space, returning ocular artifact-suppressed EEG epochs.

Before calculating the N2 and P3 peak amplitudes, z-score standardization was applied to each EEG epoch (i.e., subtracting the mean and dividing by the standard deviation of its baseline) prior to deriving the average ERP profile of Go and NoGo conditions in each session. The N2 and P3 peak amplitudes were then defined within the time intervals of 200-500 ms (i.e., a maximal negative deflection in amplitude) and 400-700 ms (i.e., a maximal positive amplitude), respectively. These time intervals were selected while taking into account that N2 and P3 could differ between individuals and groups [14]. It is worth noting that this work quantified the N2 and P3 signatures by peak amplitude instead of mean amplitude. The wide time intervals allowed the pinpointing of suitable peak candidates. Hereafter, the resultant N2 and P3 peak amplitudes were used to relate to the impulse control capability and explore differences between groups with and without pharmacological intervention. Due to the imbalanced group samples, the statistical significance of the between-session differences in behavioral and ERP outcomes was assessed by the paired sample t-test and the Wilcoxon signed-rank test for HC/PD groups and ICD group, respectively.

The EEG analytical procedures and visualization plots were performed using the open source EEGLab toolbox/scripts [39] and in-house MATLAB programs (The Mathworks, Inc., Natick, MA, USA).

\section{Results}

Figure 2 shows the behavioral outcomes of the button-pressing task, including response time (RT) to Go trials and all response errors to Go (standard errors) and NoGo (commission errors) trials. The ICD group was prone to faster responses to Go targets relative to the HC and PD groups, particularly on the $2^{\text {nd }}$ session after taking their DA medication. There was a mean RT reduction by around $9 \mathrm{~ms}$ for the ICD group ( $1^{\text {st }}$ session: $483.9 \pm 93.8 \mathrm{~ms}, 2^{\text {nd }}$ session: 474.5 $\pm 104.7 \mathrm{~ms}$ ) with respect to an 8-ms increase for the PD group and a 4-ms reduction for the HC group. However, these RT differences were not statistically significant ( $p>0.5)$. Along with such an RT outcome, only the ICD group made more response errors in the $2^{\text {nd }}$ session than in 
165

166

167

168

169

170

171

172

173

174

175

176

177

178

179

180

181

182

183

184

185

186

187

188

189

190

191

192

193

194

195

196

197

the $1^{\text {st }} \operatorname{session}\left(2^{\text {nd }}\right.$ vs. $1^{\text {st. }} 4.2 \pm 4.1$ vs. $\left.2.0 \pm 2.5 ; p=0.03\right)$. The other two groups made comparable errors $(p>0.2)$ between the two sessions.

Figure 3 compares the ERP profiles of Go and NoGo trials at the $\mathrm{Cz}$ electrode in representative subjects from the HC, PD, and ICD groups. In general, ERP images (first two rows) show how NoGo trials clearly present a N2 peak (blue strap) around 200-400 ms and a P3 peak (red strap) around 400-600 ms. The N2 and P3 signatures were relatively consistent across trials as compared to the Go counterpart. After applying synchronizing averaging to all available trials, the ERP profile (last row) exhibited N2 and P3 peaks exclusively for the NoGo condition. The Go-NoGo comparison empirically demonstrated the validity of the Go/NoGo protocol for eliciting impulse inhibition behavior and the corresponding ERP signatures of interest.

Figure 4 further presents the topographic mapping of NoGo N2 and P3 peak amplitudes and their between-session contrast at the representative electrodes for the HC, PD, and ICD groups. As can be seen, the HC group exhibited relatively stronger N2 and P3 amplitudes for both sessions as compared to the other groups. The peak distributions were maximally located at the fronto-central midline electrodes (i.e., Fz, FCz, and Cz), while the P3 distribution also expanded laterally towards F3 and F4 (as shown in Figure 4A). Furthermore, while both PD and ICD groups were administered their DA therapy after the $1^{\text {st }}$ session, only the ICD group developed noticeable deterioration in $\mathrm{N} 2$ and P3 peak amplitudes in the $2^{\text {nd }}$ session over the midline electrodes. As shown in Figure 4B, the between-session P3 amplitude difference was significant at $\mathrm{Fz}$ and $\mathrm{Cz}(p<0.04)$ and marginally significant at $\mathrm{FCz}(p=0.055)$, whereas the $\mathrm{N} 2$ contrast simply returned a tendency in decline $(0.07<p<0.16)$. On the other hand, the PD group behaved comparably to the $\mathrm{HC}$ group, barely yielding distinguishable between-session $\mathrm{N} 2$ and P3 contrasts $(0.24<p<0.94)$.

Figure 5 shows the between-session contrast in N2 and P3 peak amplitudes and their associations with ICD score. The ICD group showed more deterioration in both N2 and P3 peak amplitudes relative to the $\mathrm{HC}$ and PD groups. However, only the P3 decline in the ICD-HC comparison had statistical significance $(p<0.05)$ at Fz and Cz. Most importantly, the betweensession peak decline for both $\mathrm{N} 2$ and P3 signatures showed a relationship with ICD scores, that is, the subjects with a higher ICD score had weaker signatures after the DA treatment. Particularly, the tendency of $\mathrm{N} 2$ decline exhibited a statistical association at $\mathrm{Fz}(r=-0.28, p=$ $0.04)$ and marginally at $\mathrm{Cz}(r=-0.24, p=0.09)$. However, this association was less significant for the P3 counterpart. 


\section{Discussion}

This work contributed to explore ERP-related biomarkers that can be used to reflect DAtriggered cognitive disorders in PD patients. Furthermore, the lab-customized, cost-efficient LEGO-like EEG headset [29, 30] (regarded as non-medical grade) was successfully employed in this feasibility study. The present work found that PD patients diagnosed with ICD exhibit $\mathrm{N} 2$ and $\mathrm{P} 3$ peak amplitude deterioration upon administration of DA treatment. The ICD severity tended to modulate the N2 deterioration. Thus, the present ERP findings objectively assessed the ICD adverse effect, which can constitute a complimentary assessment to conventional scales such as clinical interviews and self-reported questionnaires performed to PD patients. The EEG wearability also facilitates neuro-monitoring in PD patients' living environments and allows to elaborate an optimal pharmacological plan while treating motor symptoms chronically. The following paragraphs discuss the integrity of the ERP outcomes and feasible refinement towards the aforementioned purpose.

The stereotypical Go/NoGo protocol was employed to arouse cognitive and motoric inhibition and to elicit the corresponding ERP signatures of N2 and P3 peaks at the frontocentral region, which appeared in response to NoGo events (i.e., successful inhibitions) [13, 25, 26]. This work implemented the 2-target visual task accordingly. The NoGo N2 (200-400 ms) and P3 (400-600 ms) components noticeably appeared (c.f., Figure 3), in line with the literature. Furthermore, the study results also showed that the PD groups (w/ and w/o ICD) exhibited weakened N2 and P3 peak amplitudes with respect to the $\mathrm{HC}$ group (c.f., Figure 4), which replicated early findings $[14,32]$. While previous studies mostly focused on EEG differences between different PD stages [33-35] or between PD vs. HC individuals [14, 31, 32], less effort has been devoted to assess PD patients against PD patients diagnosed with ICD. In order to address this issue, the present work conducted two sessions of the Go/NoGo task interleaved with the DA-pharmacological treatment for PD groups. The ICD group was the only one to exhibit a tendency to between-session decline in peak amplitude at the fronto-central midline electrodes (i.e., Fz, FCz, and Cz), particularly for the P3 counterpart (c.f., Figure 4). Beyond the between-group comparison, N2 peak deterioration was somehow modulated by ICD severity (i.e., patients' self-reported QUIP-RS scores), which was statistically remarkable at Fz and marginally at $\mathrm{Cz}$. The above comparison of PD w/ versus w/o ICD implied that the DA therapy made ICD patients vulnerable to impulse control deterioration, not only evident as behavioral manifestations (i.e., relatively faster yet mistaken responses in the $2^{\text {nd }}$ session), but also as weakened fronto-central N2 and P3 peaks. Furthermore, this work empirically 
demonstrated the potential of the cost-efficient EEG-sensing LEGO headset and of the ERP protocol and analytical framework for monitoring the impulse control capability of PD patients during pharmacological intervention. It is worth noting that the LEGO-like electrode-holder infrastructure [30] allows to unlimitedly reassemble a compact EEG headset with minimal yet informative electrodes (Fz, FCz, and $\mathrm{Cz}$ only) to the scalp. This removes redundant/irrelevant electrodes from other brain regions, improves headset wearability and comfort, and offers individual optimization for each PD patient if necessary.

Even though the above outcomes are promising, some issues should be mentioned and considered for future efforts to improve the effectiveness of the adopted protocol and framework. First, ERP waveforms are time-locked and phase-locked weak electrical potentials to stimulation events. By applying the synchronizing averaging to repetitively collected trials of the same task, the ERP components of interest (e.g., N2 and P3 signatures) can be revealed, since EEG background activity (i.e., concurrent to the engaged task) and/or accompanying random noises can be greatly alleviated at the same time. As such, the number of collected trials and the extent of artifact contamination are two critical factors for the signatures' signal-tonoise ratio (SNR). However, the present work was a user-friendly EEG experiment for elderly subjects (typically above $59 \mathrm{yrs}$ ). We, therefore, reduced sessions to around 30 minutes and mounted the dry electrodes over the LEGO headset. As such, each single session only collected 72 NoGo trials per subject, which had an infrequent occurrence of $30 \%$. The retained trials were even less after noisy trial removal ( $\sim 9 \%$ removed on average). The limited trials, thus, inevitably downgraded the N2 and P3 SNR for certain subjects. This may explain in part the noticeable within-group and between-group variability at the fronto-central electrodes (c.f., Figures 4B and 5). Future efforts should incorporate advanced artifact removal frameworks or spatial filtering frameworks [40-43] to improve SNR given the number of EEG trials collected with this challenging recording setting. On the other hand, the Go/NoGo protocol configuration, in particular trial pace (i.e., stimulus-stimulus interval) and the probability of NoGo trials, has been reported to affect the capability to elicit prepotent motor activity and probe inhibitory control [26]. By varying color-square cues and their presence, a fast-paced trial (1.5 seconds) with a rare NoGo occurrence $(20 \%)$ reliably increased prepotent motor activity and inhibitory control-related activity. Conversely, a slow-pace version ( $>4$ seconds) and/or equiprobable Go/NoGo events (50\%) considerately compromised the frontocentral N2 and P3 amplitudes [26]. Even though the present work (stimulus-stimulus interval: 1.5-2.5 seconds, NoGo probability: $30 \%$ ) considerately complied with the fast and rare configurations, future studies 
264 can aim to include configurations that resemble the former to evaluate whether the resultant between-session N2 and P3 components can be further amplified.

\section{Limitations}

267

268

269

270

271

272

273

274

275

276

277

278

279

280

281

282

283

284

285

286

287

288

289

290

291

292

294

293 QUIP-RS: Questionnaire for impulsive-compulsive disorders in PD-rating scale

The present work has some limitations. The existence of ICD-related ERP biomarkers was empirically demonstrated in a relatively small number of ICD subjects. The biomarkers' generalizability has to be tested in a larger population in future. Another limiting factor is that this work performed a single-day EEG recording and analysis. That is, each recruited subject participated in the Go/NoGo protocol with and without DA treatment only once. However, intra-individual differences in task-related EEG activities may present ecologically on a daily basis [44-47]. Several behavioral and psychological states such as attention, stress, anxiety and/or sleep quality may contribute to the above EEG non-stationarity. Effectively alleviating non-stationarity is still an open challenge [48-50], and, thus, the ERP-marker's robustness has to be elucidated over repeated measurements interspaced in the chronic pharmacological plans.

\section{Conclusion}

This work empirically demonstrated that the customized low-cost LEGO-like EEG headset enabled to extract ERP neuromarkers for the objective assessment of ICD in patients with PD during DA treatment. The ERP evidence may provide complementary information to behavioral evaluation, which is conventionally used to diagnose the ICD adverse effect.

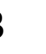

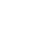

\section{Abbreviations}

DA: Dopamine agonist

EEG: Electroencephalogram

ERP: Event-related potential

HC: Healthy control

ICD: Impulse control disorder

ICA: Independent component analysis

PD: Parkinson's disease

RT: Response time 
295 Ethics approval and consent to participate

296 The Institutional Review Board of Kaohsiung Chang Gung Memorial Hospital approved all the

297 procedures. Written informed consent was obtained from all the participants.

298

299 Consent for publication

300 Not applicable.

301

302

Availability of data and materials

303

The datasets generated and/or analyzed during the current study are available from the corresponding author on reasonable request.

305

306

\section{Competing interests}

307 The authors declare that they have no competing interests.

308

309 Funding

310 This work was supported in part by Collaborative Research Project of National Sun Yat-sen

311 University and Kaohsiung Chang Gung Memorial Hospital, Taiwan, under Grant

312 CMRPG8I0371.

313

314 Authors' contributions

315 YPL conceived the experiments and the data analysis, analyzed the data, interpreted the results, and wrote the manuscript. HYL prepared and performed the experiment, analyzed the data, and wrote the manuscript. YSC, CHL, YRW, and YYC contributed to the design of the study, managed the subjects, and reviewed the manuscript. WCL contributed to the design of the study, managed the subjects, interpreted the results, and reviewed the manuscript. All authors read and approved the final manuscript.

\section{Acknowledgements}

323 The authors thank Kai-Chiang Chuang for his contribution in the development and validation of EEG amplifier. 


\section{References}

1. Lotharius J, Brundin P: Pathogenesis of Parkinson's disease: dopamine, vesicles and alphasynuclein. Nat Rev Neurosci 2002, 3:932-942.

2. Brown RG, Marsden CD: Cognitive function in Parkinson's disease: from description to theory. Trends in neurosciences 1990, 13:21-29.

3. Lee E-Y, Cowan N, Vogel EK, Rolan T, Valle-Inclán F, Hackley SA: Visual working memory deficits in patients with Parkinson's disease are due to both reduced storage capacity and impaired ability to filter out irrelevant information. Brain 2010, 133:2677-2689.

4. Weintraub D, Koester J, Potenza MN, Siderowf AD, Stacy M, Voon V, Whetteckey J, Wunderlich GR, Lang AE: Impulse control disorders in Parkinson disease: a cross-sectional study of 3090 patients. Archives of neurology 2010, 67:589-595.

5. Seedat S, Kesler S, Niehaus DJ, Stein DJ: Pathological gambling behaviour: emergence secondary to treatment of Parkinson's disease with dopaminergic agents. Depress Anxiety 2000, 11:185-186.

6. Driver-Dunckley E, Samanta J, Stacy M: Pathological gambling associated with dopamine agonist therapy in Parkinson's disease. Neurology 2003, 61:422-423.

7. Weintraub D, David AS, Evans AH, Grant JE, Stacy M: Clinical spectrum of impulse control disorders in Parkinson's disease. Movement disorders : official journal of the Movement Disorder Society 2015, 30:121-127.

8. Voon V, Gao J, Brezing C, Symmonds M, Ekanayake V, Fernandez H, Dolan RJ, Hallett M: Dopamine agonists and risk: impulse control disorders in Parkinson's disease. Brain : a journal of neurology 2011, 134:1438-1446.

9. Cilia R, van Eimeren T: Impulse control disorders in Parkinson's disease: seeking a roadmap toward a better understanding. Brain Structure and Function 2011, 216:289-299.

10. Hudak J, Blume F, Dresler T, Haeussinger FB, Renner TJ, Fallgatter AJ, Gawrilow C, Ehlis A-C: Near-Infrared Spectroscopy-Based Frontal Lobe Neurofeedback Integrated in Virtual Reality Modulates Brain and Behavior in Highly Impulsive Adults. Front Hum Neurosci 2017, 11.

11. Goldstein M, Brendel G, Tuescher O, Pan H, Epstein J, Beutel M, Yang Y, Thomas K, Levy K, Silverman M, et al: Neural substrates of the interaction of emotional stimulus processing and motor inhibitory control: An emotional linguistic go/no-go fMRI study. Neuroimage 2007, 36:1026-1040.

12. Palermo S, Morese R, Zibetti M, Dematteis F, Sirgiovanni S, Stanziano M, Valentini MC, Lopiano L: Impulse control disorder and response-inhibition alterations in Parkinson's disease. A rare case of totally absent functionality of the medial-prefrontal cortex and review of literature. $J$ Adv Res 2017, 8:713-716.

13. Baumeister S, Hohmann S, Wolf I, Plichta MM, Rechtsteiner S, Zangl M, Ruf M, Holz N, Boecker $\mathrm{R}$, Meyer-Lindenberg A, et al: Sequential inhibitory control processes assessed through simultaneous EEG-fMRI. Neuroimage 2014, 94:349-359.

14. Bokura H, Yamaguchi S, Kobayashi S: Event-related potentials for response inhibition in Parkinson's disease. Neuropsychologia 2005, 43:967-975.

15. McMackin R, Bede P, Pender N, Hardiman O, Nasseroleslami B: Neurophysiological markers of network dysfunction in neurodegenerative diseases. NeuroImage: Clinical 2019, 22:101706.

16. Valentin O, Ducharme M, Cretot-Richert G, Monsarrat-Chanon H, Viallet G, Delnavaz A, Voix J: Validation and Benchmarking of a Wearable EEG Acquisition Platform for Real-World Applications. Ieee T Biomed Circ S 2019, 13:103-111.

17. Melnik A, Legkov P, Izdebski K, Karcher SM, Hairston WD, Ferris DP, Konig P: Systems, Subjects, Sessions: To What Extent Do These Factors Influence EEG Data? Front Hum Neurosci 2017, 11.

18. Kam JWY, Griffin S, Shen A, Patel S, Hinrichs H, Heinze HJ, Deouell LY, Knight RT: Systematic comparison between a wireless EEG system with dry electrodes and a wired EEG system with wet electrodes. Neuroimage 2019, 184:119-129.

19. Nakanishi M, Wang YT, Jung TP, Zao JK, Chien YY, Diniz A, Daga FB, Lin YP, Wang YJ, Medeiros FA: Detecting Glaucoma With a Portable Brain-Computer Interface for Objective Assessment 
of Visual Function Loss. Jama Ophthalmol 2017, 135:550-557.

20. Mullen TR, Kothe CAE, Chi YM, Ojeda A, Kerth T, Makeig S, Jung T, Cauwenberghs G: Realtime neuroimaging and cognitive monitoring using wearable dry EEG. IEEE Transactions on Biomedical Engineering 2015, 62:2553-2567.

21. Cruz-Garza JG, Brantley JA, Nakagome S, Kontson K, Megjhani M, Robleto D, Contreras-Vidal JL: Deployment of Mobile EEG Technology in an Art Museum Setting: Evaluation of Signal Quality and Usability. Front Hum Neurosci 2017, 11.

22. Oliveira AS, Schlink BR, Hairston WD, König P, Ferris DP: Proposing Metrics for Benchmarking Novel EEG Technologies Towards Real-World Measurements. Front Hum Neurosci 2016, 10.

23. Kerick SE, Oie KS, McDowell K: Assessment of EEG Signal Quality in Motion Environments. Army Research Laboratory, Report ARL-TR-4866 2009.

24. Silva LWD, Cartier C, Cheniaux E, Novis F, Silveira LA, Cavaco PA, da Silva RD, Batista WA, Tanaka GK, Gongora M, et al: Electrical mapping in bipolar disorder patients during the oddball paradigm. J Psychiatr Res 2016, 72:64-71.

25. Zamorano F, Billeke P, Hurtado JM, López V, Carrasco X, Ossandón T, Aboitiz F: Temporal Constraints of Behavioral Inhibition: Relevance of Inter-stimulus Interval in a Go-Nogo Task. Plos One 2014, 9:e87232.

26. Wessel JR: Prepotent motor activity and inhibitory control demands in different variants of the go/no-go paradigm. Psychophysiology 2017.

27. Liu ZX, Lishak V, Tannock R, Woltering S: Effects of working memory training on neural correlates of Go/Nogo response control in adults with ADHD: A randomized controlled trial. Neuropsychologia 2017, 95:54-72.

28. Dong GH, Zhou H, Zhao X: Impulse inhibition in people with Internet addiction disorder: Electrophysiological evidence from a Go/NoGo study (vol 485, pg 138, 2010). Neurosci Lett 2011, 496:200-200.

29. Chuang KC, Lin YP: Cost-Efficient, Portable, and Custom Multi-Subject Electroencephalogram Recording System. IEEE Access 2019, 7:56760-56769.

30. Lin YP, Chen TY, Chen WJ: Cost-efficient and Custom Electrode-holder Assembly Infrastructure for EEG Recordings. Sensors-Basel 2019, 19.

31. Chu C, Wang X, Cai L, Zhang L, Wang J, Liu C, Zhu X: Spatiotemporal EEG microstate analysis in drug-free patients with Parkinson's disease. NeuroImage: Clinical 2020, 25:102132.

32. Pulvermüller F, Lutzenberger W, Müller V, Mohr B, Dichgans J, Birbaumer N: P3 and contingent negative variation in Parkinson's disease. Electroen Clin Neuro 1996, 98:456-467.

33. Shah SAA, Zhang L, Bais A: Dynamical system based compact deep hybrid network for classification of Parkinson disease related EEG signals. Neural Networks 2020, 130:75-84.

34. Betrouni N, Delval A, Chaton L, Defebvre L, Duits A, Moonen A, Leentjens AFG, Dujardin K: Electroencephalography-based machine learning for cognitive profiling in Parkinson's disease: Preliminary results. Movement disorders : official journal of the Movement Disorder Society 2019, 34:210-217.

35. Uslu A, Ergen M, Demirci H, Lohmann E, Hanagasi H, Demiralp T: Event-related potential changes due to early-onset Parkinson's disease in parkin (PARK2) gene mutation carriers and non-carriers. Clin Neurophysiol 2020, 131:1444-1452.

36. Weintraub D, Mamikonyan E, Papay K, Shea JA, Xie SX, Siderowf A: Questionnaire for Impulsive-Compulsive Disorders in Parkinson's Disease-Rating Scale. Movement disorders : official journal of the Movement Disorder Society 2012, 27:242-247.

37. Yang SY, Lin YP: Validating a LEGO-Like EEG Headset for a Simultaneous Recording of Wetand Dry-Electrode Systems During Treadmill Walking. In 42nd Annual International Conference of the IEEE Engineering in Medicine \& Biology Society (EMBC); 20-24 July 2020. 2020: 40554058.

38. Jung TP, Makeig S, Humphries C, Lee TW, McKeown MJ, Iragui V, Sejnowski TJ: Removing electroencephalographic artifacts by blind source separation. Psychophysiology 2000, 37:163178.

39. Delorme A, Makeig S: EEGLAB: an open source toolbox for analysis of single-trial EEG dynamics including independent component analysis. $J$ Neurosci Meth 2004, 134:9-21.

40. Chang C, Hsu S, Pion-Tonachini L, Jung T: Evaluation of Artifact Subspace Reconstruction for 
Automatic Artifact Components Removal in Multi-channel EEG Recordings. Ieee T Bio-Med Eng 2019:1-1.

41. $\mathrm{Hu} \mathrm{L}$, Mouraux $\mathrm{A}, \mathrm{Hu} \mathrm{Y}$, Iannetti GD: A novel approach for enhancing the signal-to-noise ratio and detecting automatically event-related potentials (ERPs) in single trials. Neuroimage 2010, 50:99-111.

42. Bufacchi RJ, Magri C, Novembre G, Iannetti GD: Local Spatial Analysis (LSA): An easy-to-use adaptive spatial EEG filter. $J$ Neurophysiol, 0:null.

43. Aydarkhanov R, Uscumlic M, Chavarriaga R, Gheorghe L, Millan JD: Spatial covariance improves BCI performance for late ERPs components with high temporal variability. Journal of Neural Engineering 2020, 17.

44. Arvaneh M, Guan CT, Ang KK, Quek C: EEG Data Space Adaptation to Reduce Intersession Nonstationarity in Brain-Computer Interface. Neural Comput 2013, 25:2146-2171.

45. Yin Z, Zhang JH: Cross-session classification of mental workload levels using EEG and an adaptive deep learning model. Biomedical Signal Processing and Control 2017, 33:30-47.

46. Garcia L, Zak M, Grenier C, Hanrio S, Henry D, Randriamanantena R, Semal C, Andre JM, Lespinet-Najib V, Ron-Angevin R: Is Stress State an Important Factor in the BCI-P300 Speller Performance? In Advances in Computational Intelligence; 2019//; Cham. Edited by Rojas I, Joya G, Catala A. Springer International Publishing; 2019: 442-454.

47. Shen YW, Lin YP: Challenge for Affective Brain-Computer Interfaces: Non-stationary Spatiospectral EEG Oscillations of Emotional Responses. Front Hum Neurosci 2019, 13:366-366.

48. Wan Z, Yang R, Huang M, Zeng N, Liu X: A review on transfer learning in EEG signal analysis. Neurocomputing 2021, 421:1-14.

49. Kaltenstadler S, Nakajima S, Müller K, Samek W: Wasserstein Stationary Subspace Analysis. IEEE Journal of Selected Topics in Signal Processing 2018, 12:1213-1223.

50. Lin YP: Constructing a Personalized Cross-Day EEG-Based Emotion-Classification Model Using Transfer Learning. IEEE Journal of Biomedical and Health Informatics 2020, 24:12551264. 


\section{Figure captions:}

Figure 1. Experiment protocol and EEG recording setup. (A) The designed two-target visual Go/NoGo task, (B) the 8-channel EEG montage, and (C) a snapshot of an EEG experiment with the assembled LEGO-like headset.

Figure 2. Behavioral results of the Go/NoGo task. (A) Response time (ms) for Go trials. (B) Response errors for Go and NoGo trials.

Figure 3. ERP images and profiles at $\mathrm{Cz}$ from representative subjects of $\mathrm{HC}, \mathrm{PD}$, and ICD groups. The NoGo trial (red trace) corresponds to N2 and P3 peaks around 200-400 ms and 400-600 ms, respectively, with respect to Go trials (blue trace).

Figure 4. Comparative NoGo $\mathrm{N} 2$ and $\mathrm{P} 3$ signatures between the $1^{\text {st }}$ and $2^{\text {nd }}$ sessions and their contrast. Only PD and ICD group underwent DA treatment right after the $1^{\text {st }}$ session. (A) Topographic mapping of peak amplitudes over the adopted 8-channel montage. The color was normalized according to the amplitudes across groups. (B) ERP profiles and peak amplitude distributions. * indicates statistically significant between-session difference $(p<0.05)$.

Figure 5. Between-session contrast of NoGo N2 and P3 peak amplitudes and their associations with ICD scores at the representative electrodes. Circles at the right side of each subplot represent the outcome for each individual, whereas gray lines depict the linear relationships between the peak differences and ICD scores assessed by linear regression analysis. * indicates the statistically significant between-group difference $(p<0.05)$. 
Figure 1

(A)

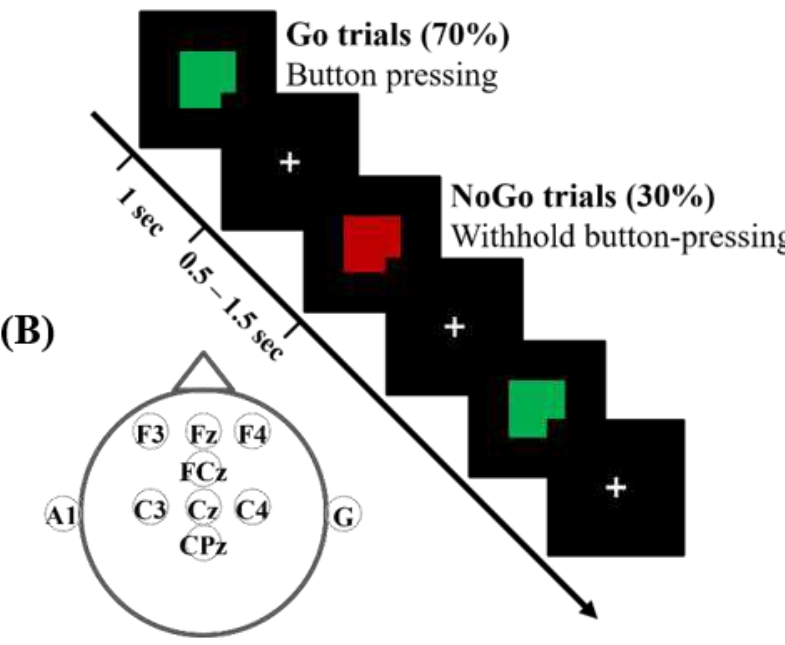

(C)

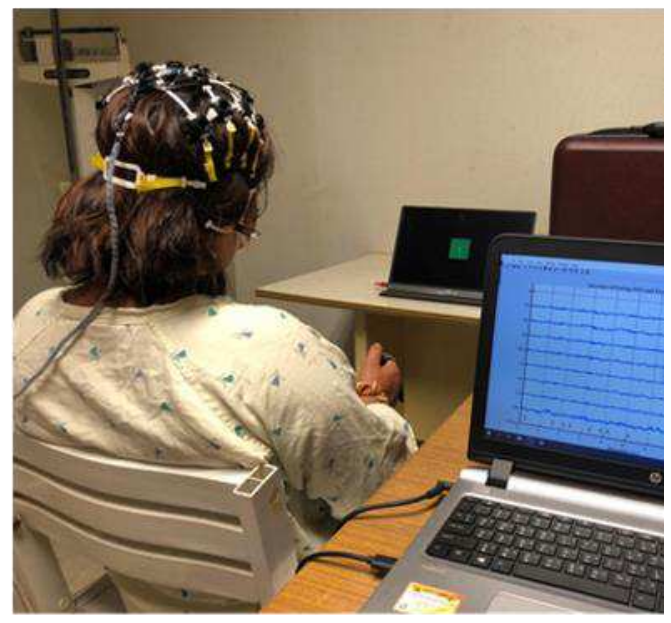

Figure 2

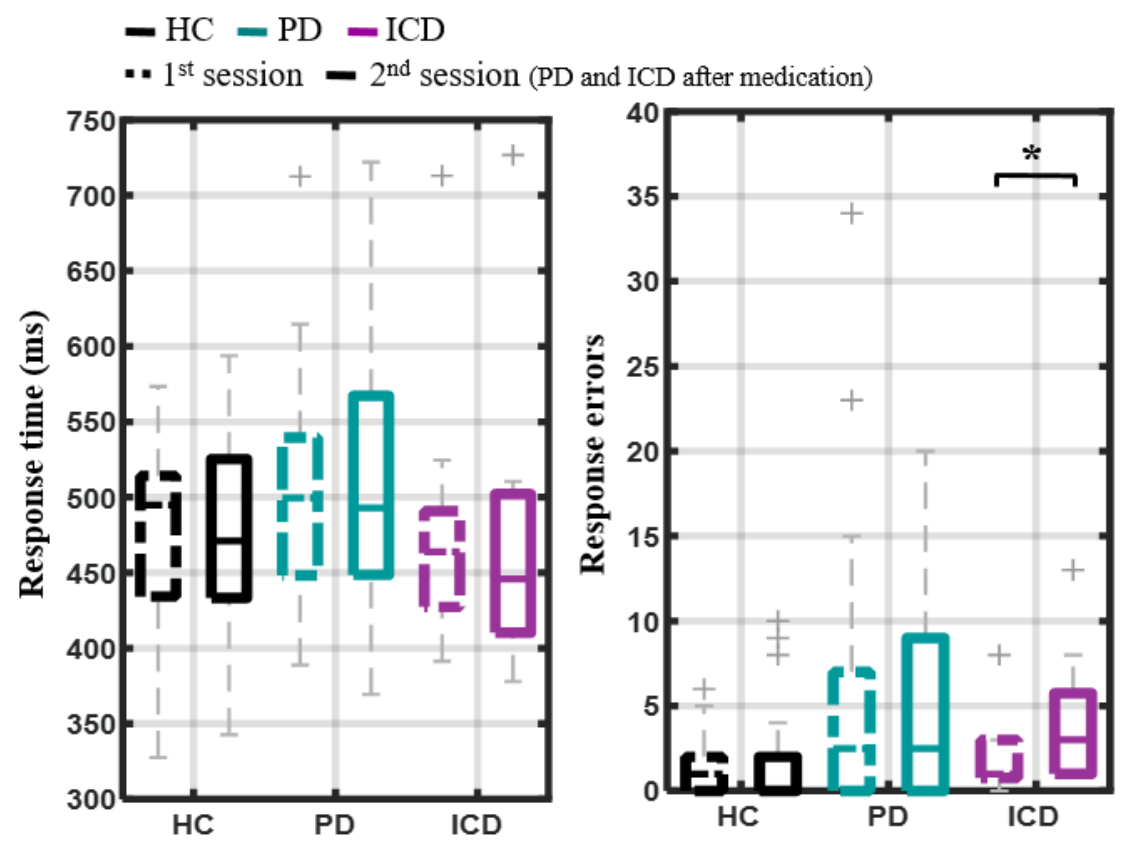


Figure 3
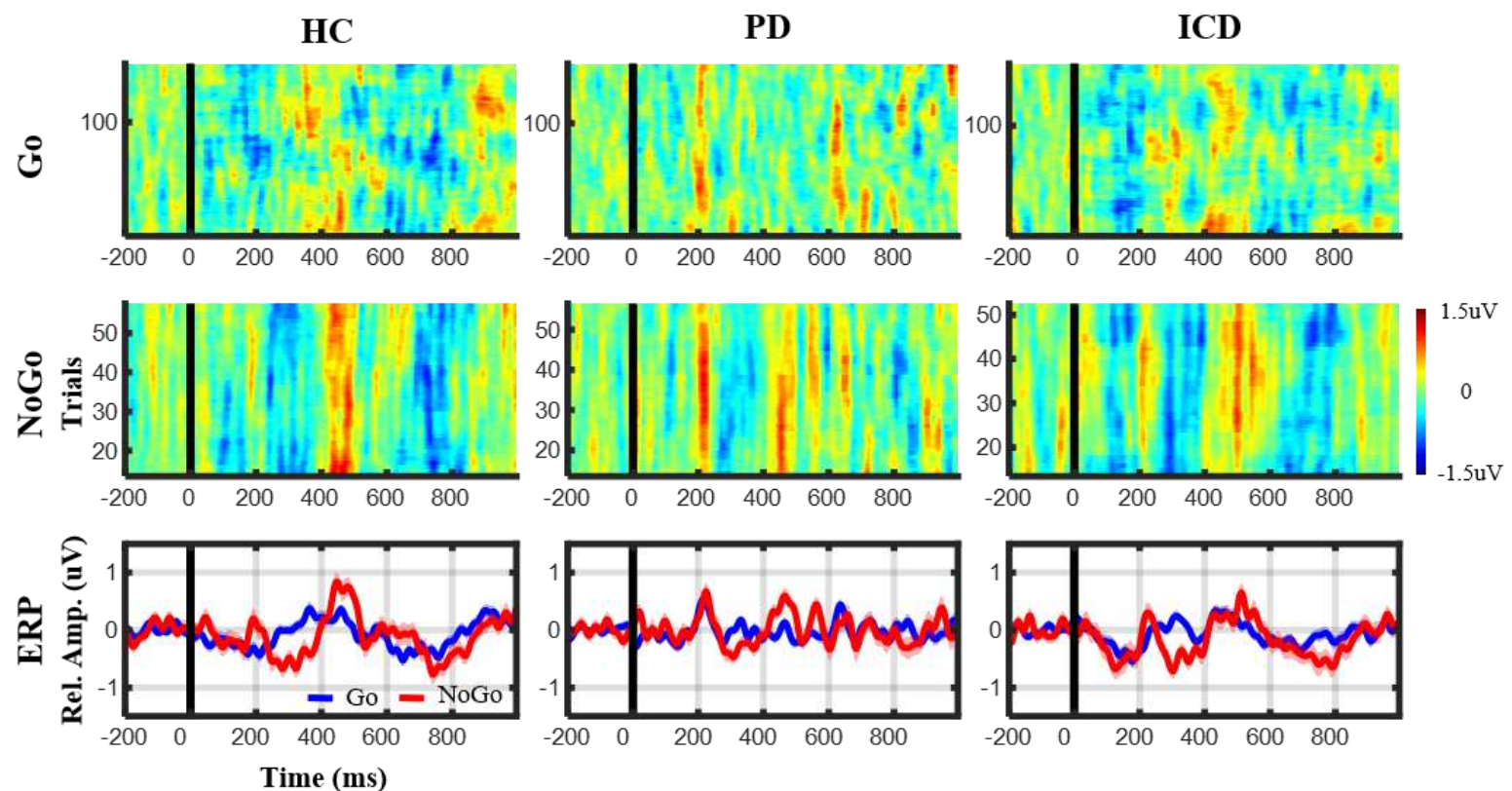
Figure 4A

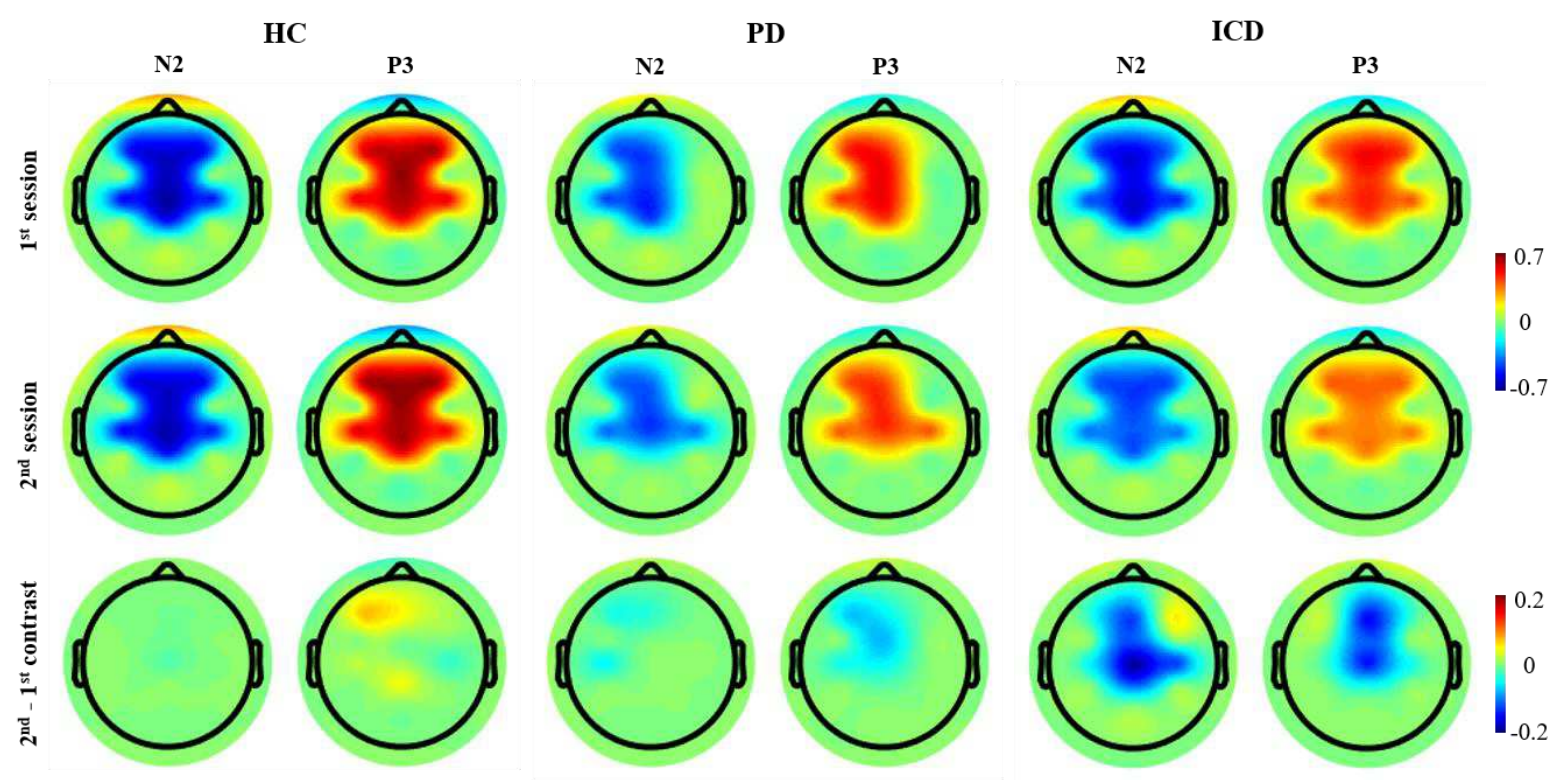

Figure 4B
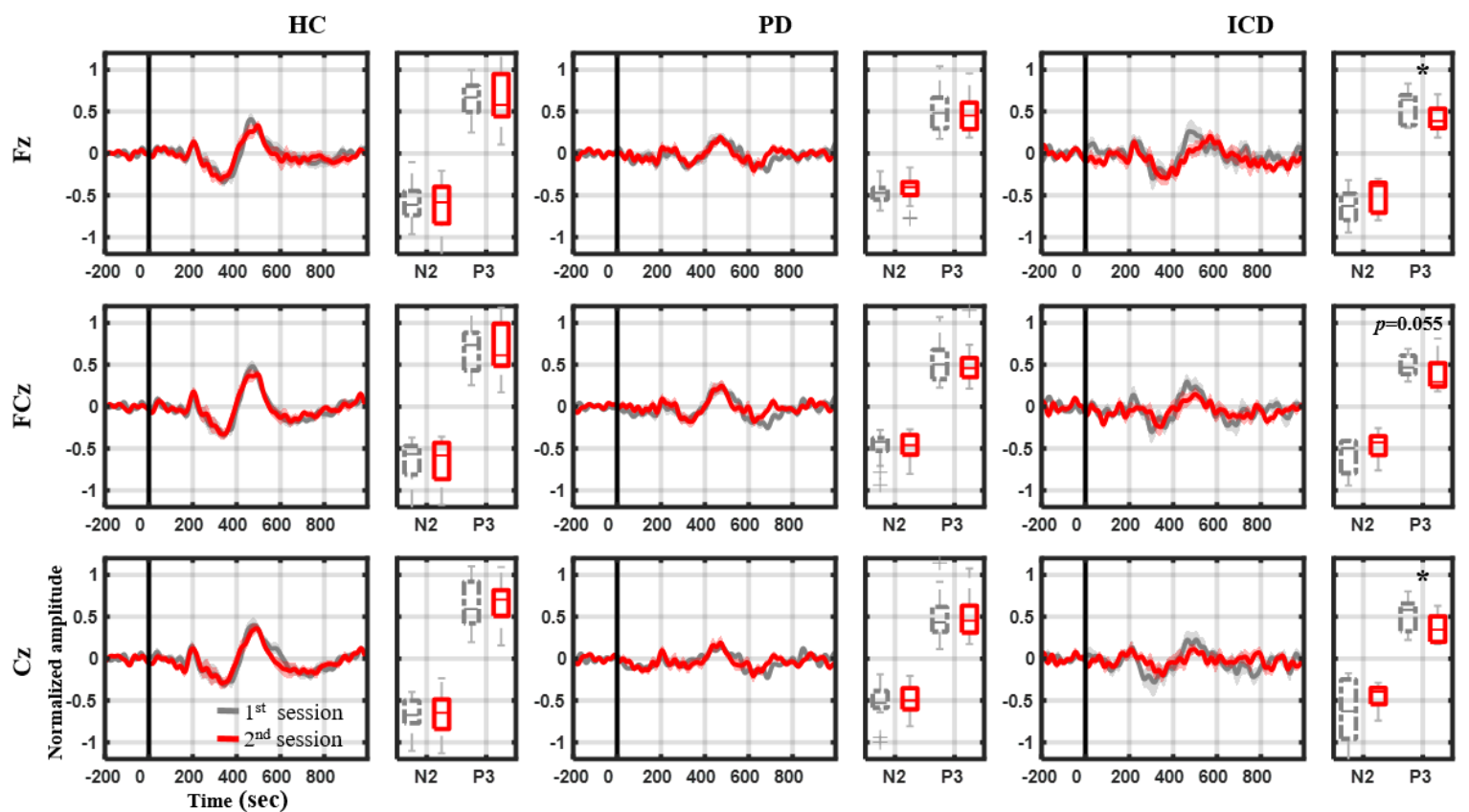
Figure 5
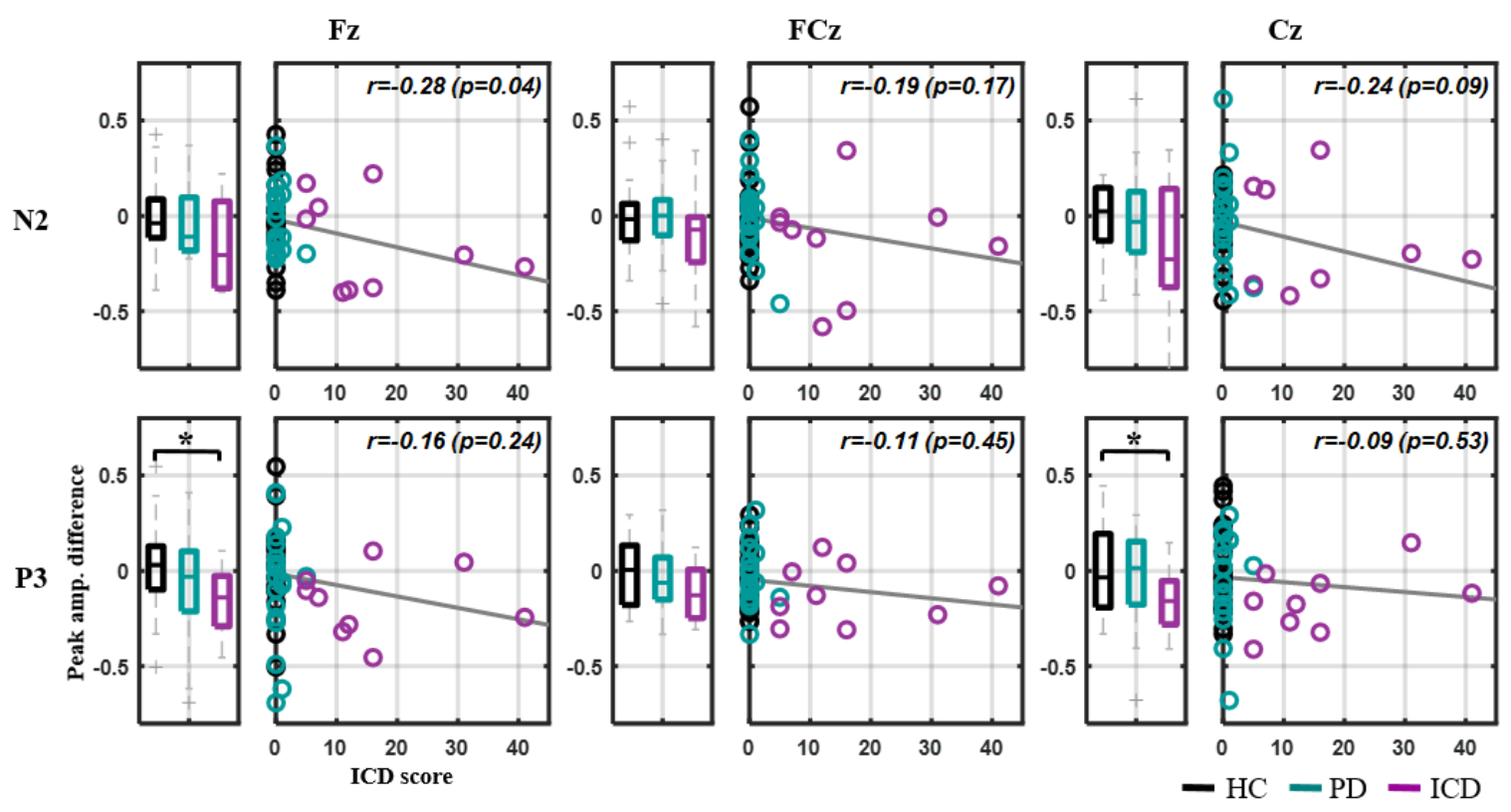
(A)

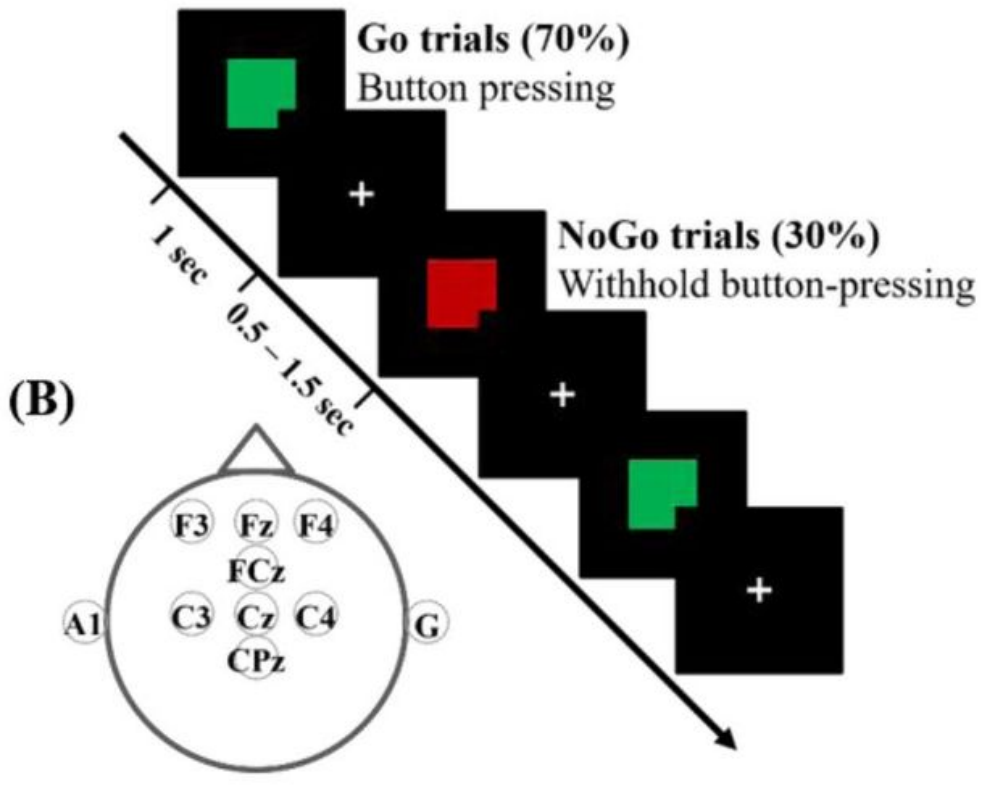

(C)

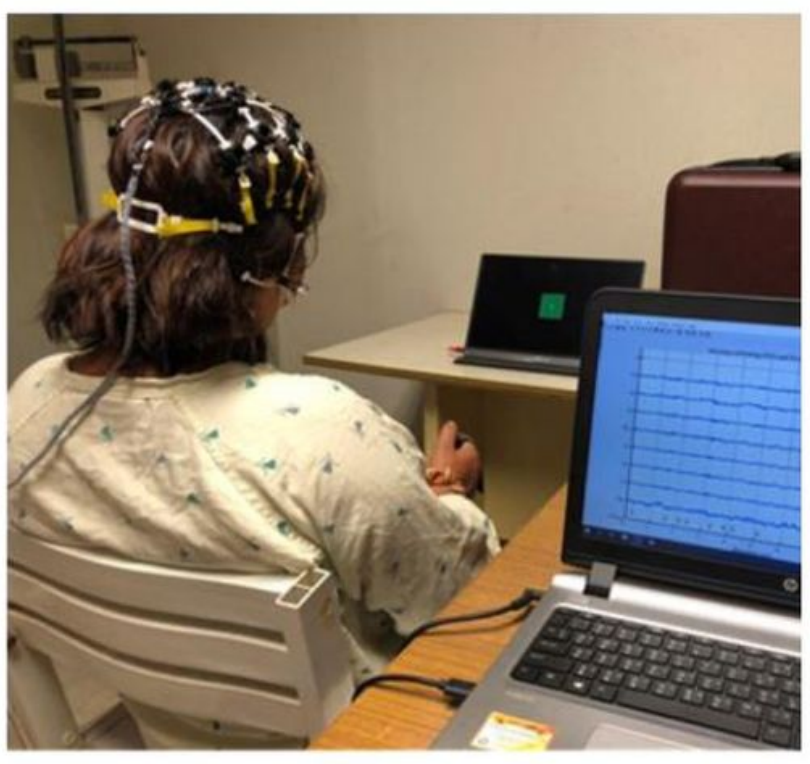

\section{Figure 1}

Experiment protocol and EEG recording setup. (A) The designed two-target visual Go/NoGo task, (B) the 8-channel EEG montage, and (C) a snapshot of an EEG experiment with the assembled LEGO-like headset. 


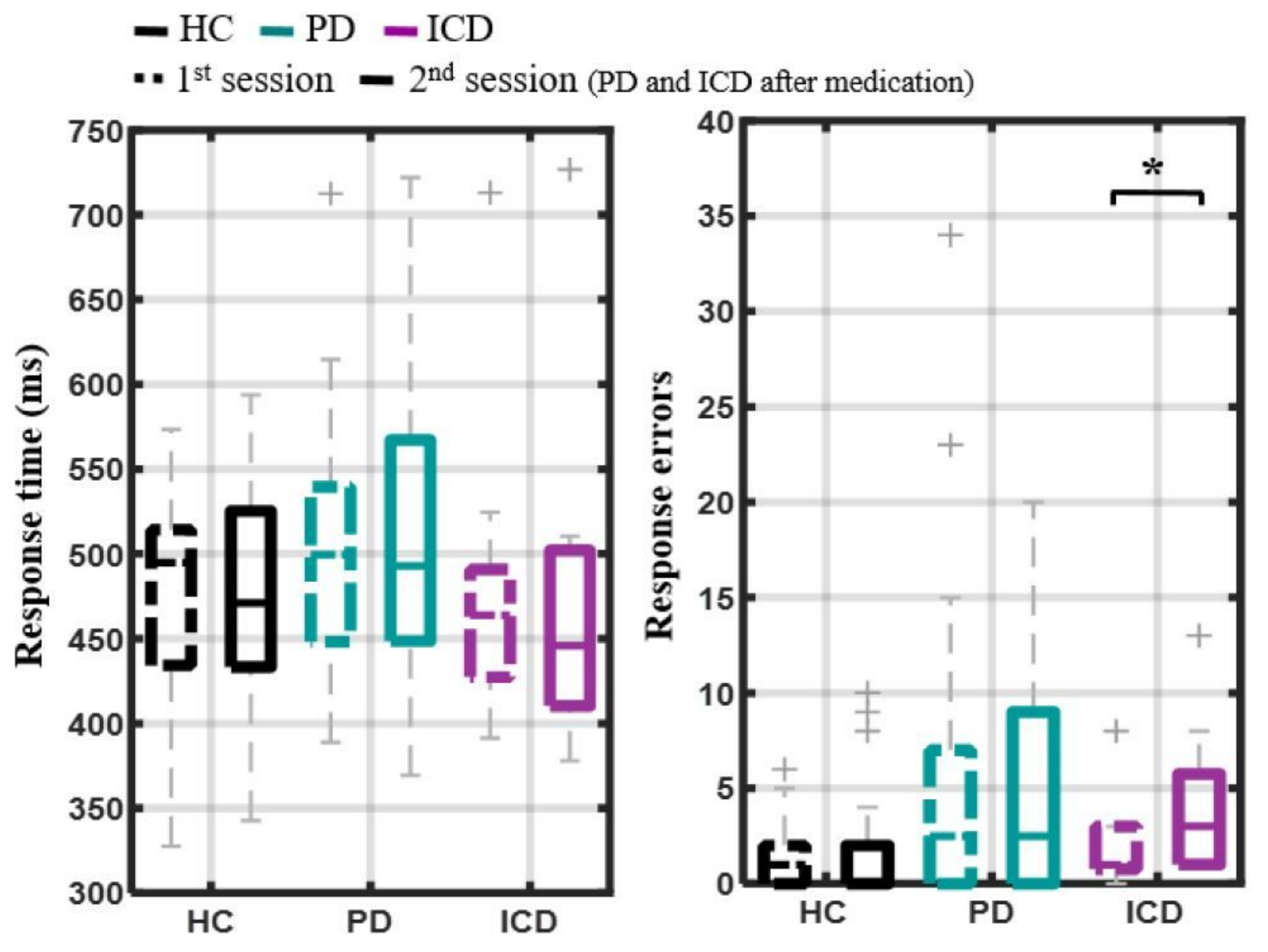

Figure 2

Behavioral results of the Go/NoGo task. (A) Response time (ms) for Go trials. (B) Response errors for Go and NoGo trials. 
HC
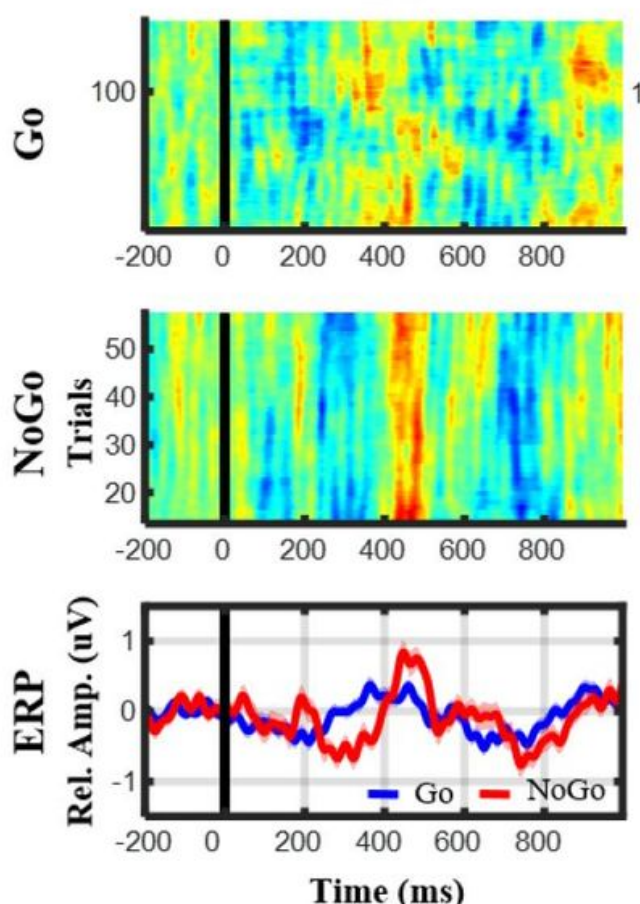

PD
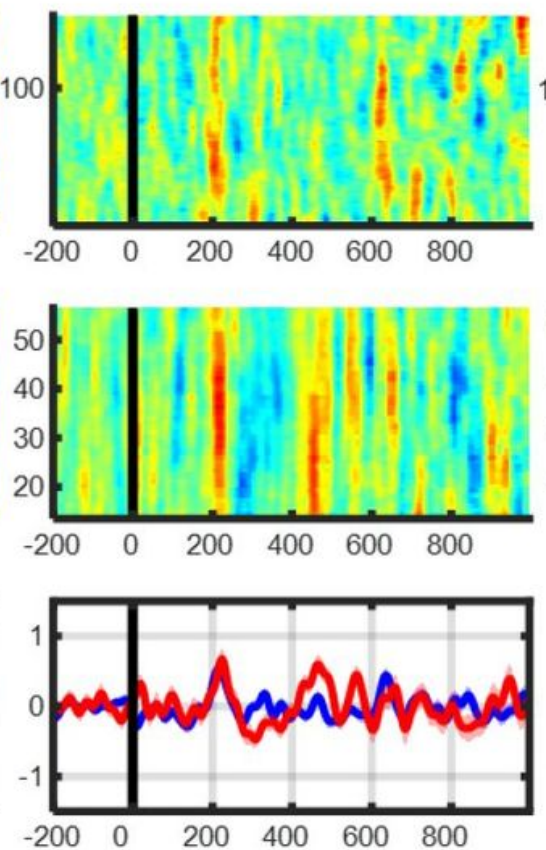

ICD
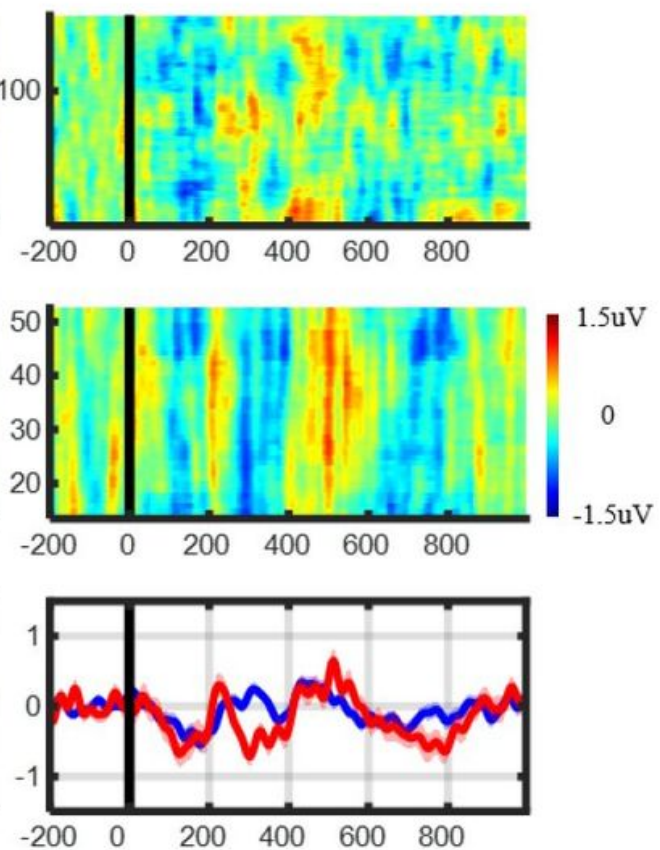

Figure 3

ERP images and profiles at $\mathrm{Cz}$ from representative subjects of HC, PD, and ICD groups. The NoGo trial (red trace) corresponds to N2 and P3 peaks around 200-400 ms and 400-600 ms, respectively, with respect to Go trials (blue trace). 


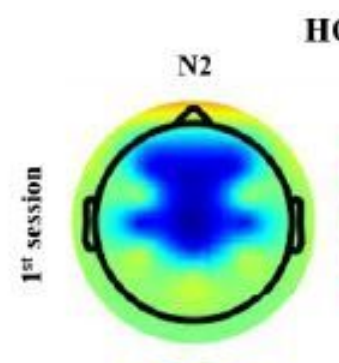

HC

PD
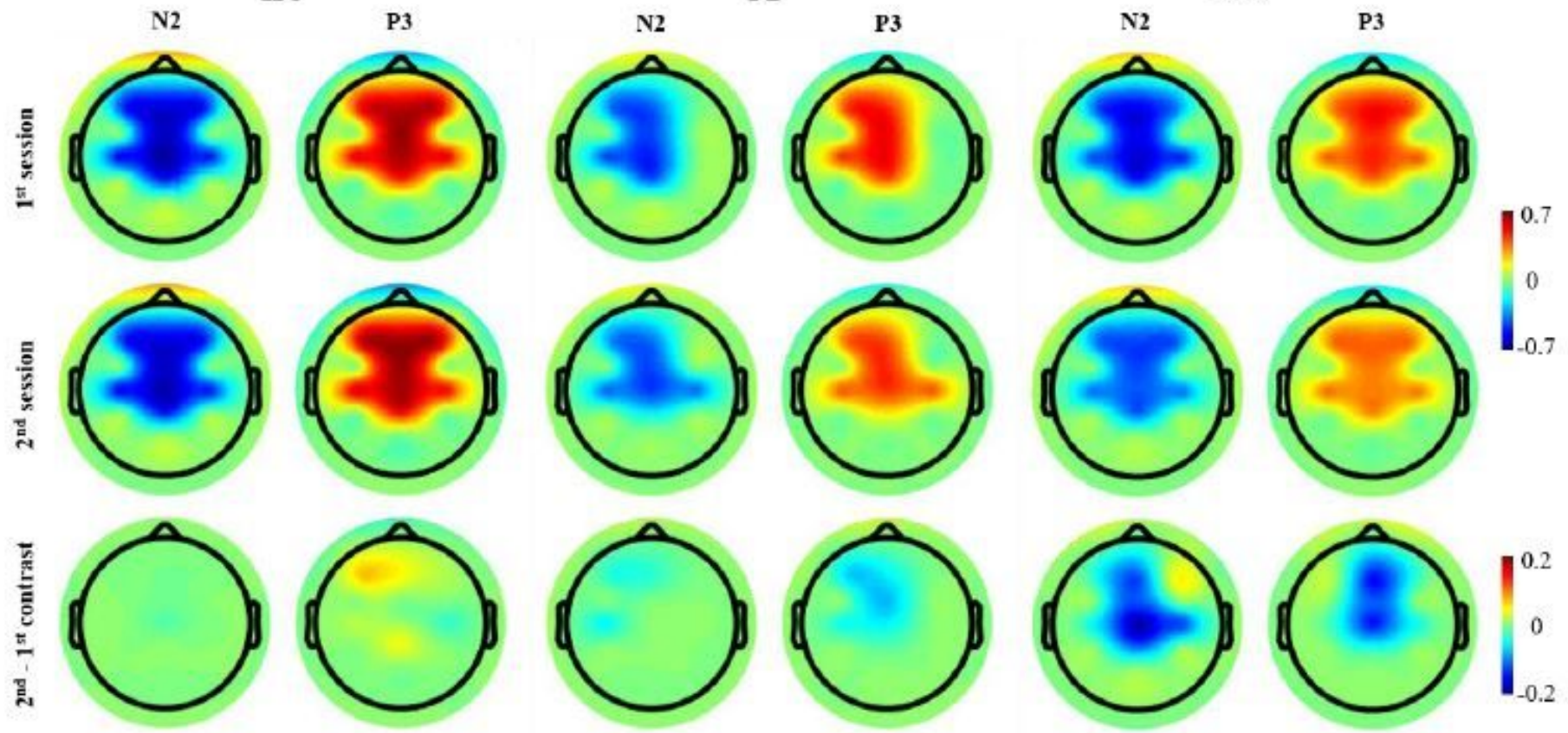

Figure 4B

HC
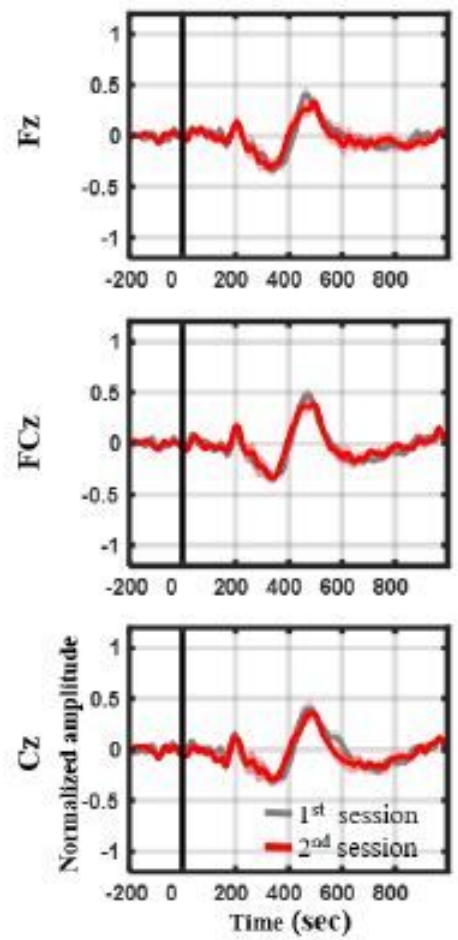

PD
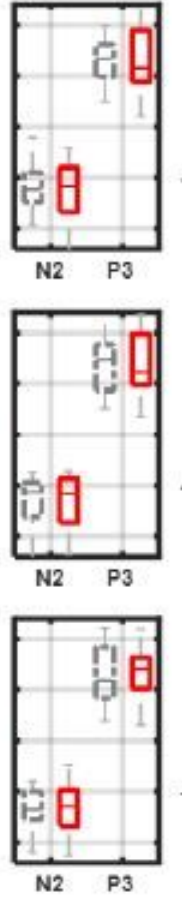
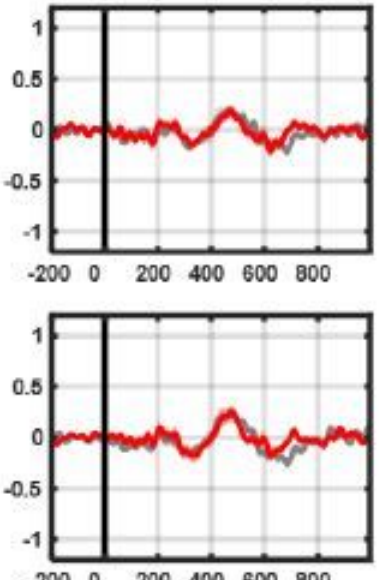

$-200 \quad 0 \quad 200 \quad 400600 \quad 800$

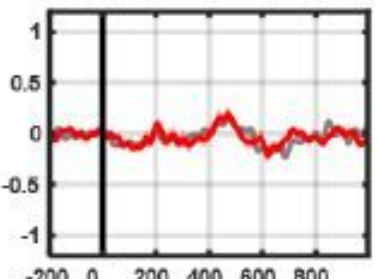

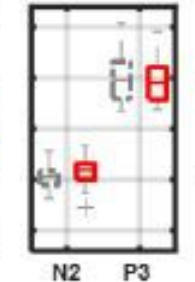
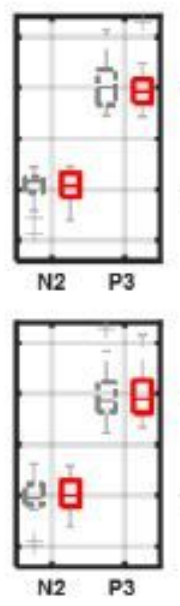

ICD
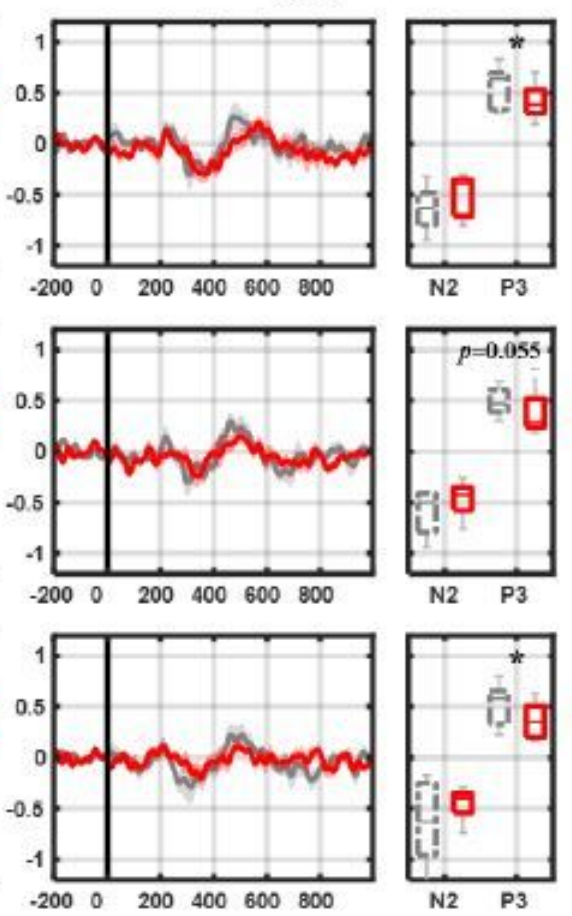

Figure 4

Comparative NoGo N2 and P3 signatures between the 1st and 2nd sessions and their contrast. Only PD and ICD group underwent DA treatment right after the 1st session. (A) Topographic mapping of peak amplitudes over the adopted 8-channel montage. The color was normalized according to the amplitudes across groups. (B) ERP profiles and peak amplitude distributions. * indicates statistically significant between-session difference $(p<0.05)$. 
Fz
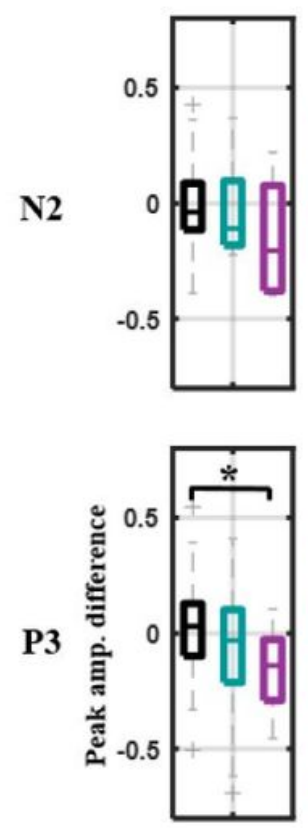
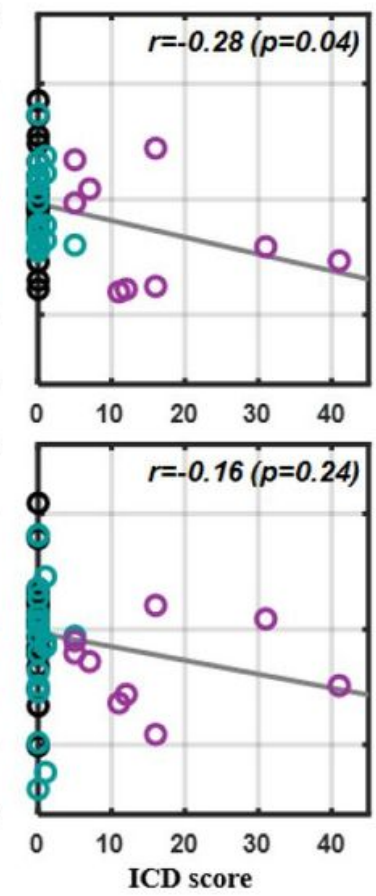

$\mathrm{FCz}$
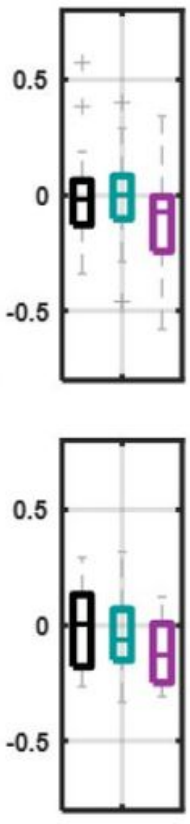
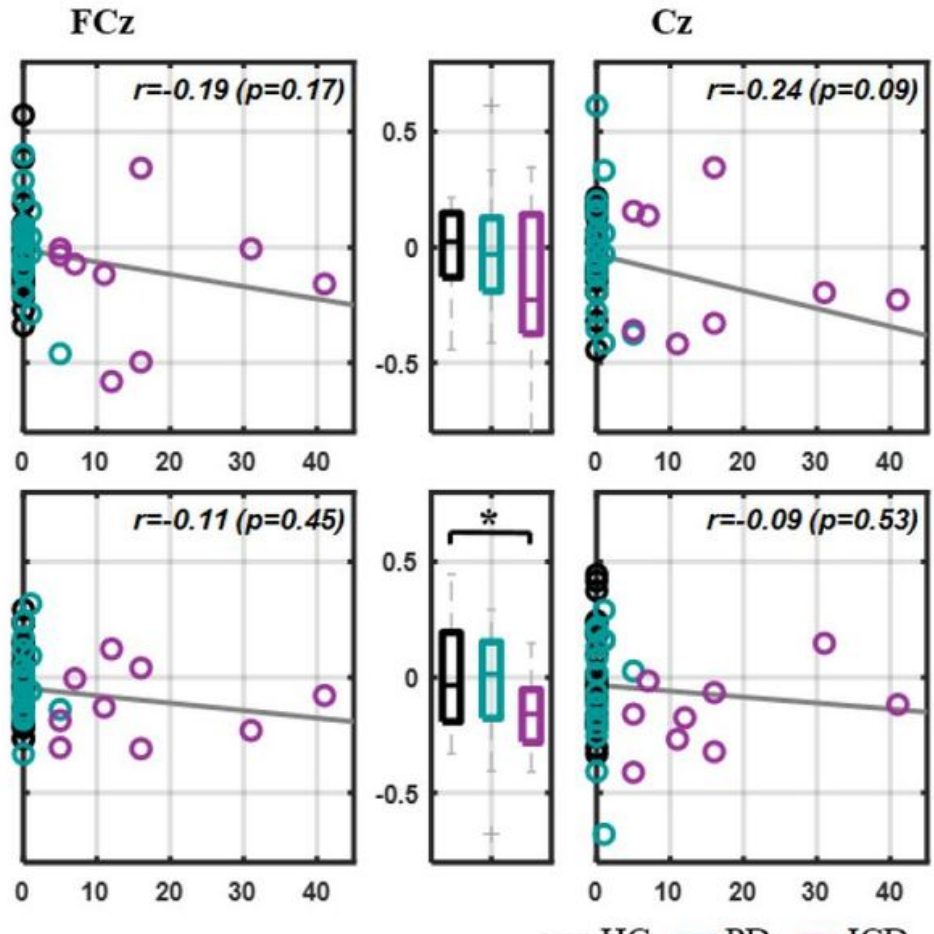
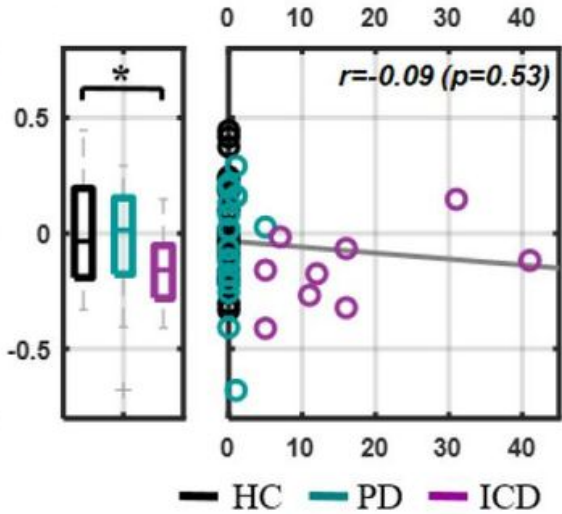

Figure 5

Between-session contrast of NoGo N2 and P3 peak amplitudes and their associations with ICD scores at the representative electrodes. Circles at the right side of each subplot represent the outcome for each individual, whereas gray lines depict the linear relationships between the peak differences and ICD scores assessed by linear regression analysis. * indicates the statistically significant between-group difference $(p<0.05)$. 